\title{
Microhimenópteros coletados de pupas procedentes de fezes de gado bovino em três propriedades rurais do sul do Estado de Goiás, Brasil
}

\author{
Microhimenopterous collected from pupae proceeding feces of cattle in \\ three rural properties in the south of the State of Goiás, Brazil
}

\author{
Carlos Henrique Marchiori ${ }^{1 *}$; Otacílio Moreira Silva Filho²; Márcio de Paula Borges ${ }^{3}$
}

\begin{abstract}
Resumo
Este estudo teve como objetivo determinar as espécies de microhimenópteros parasitóides em pupas de Diptera presentes em fezes bovinas, coletadas nos municípios de Itumbiara, Cachoeira Dourada, e Panamá, GO, no período de janeiro de 1998 a junho de 2004. As pupas dos dípteros foram isoladas pelo método de flotação, individualizadas em cápsulas de gelatina até a emergência das moscas e/ou dos seus parasitóides. A porcentagem total de parasitismo observada foi de 7,36\%. As espécies mais freqüentes foram: Spalangia drosophilae Ashmead (Hymenoptera: Pteromalidae) com 30,8\% e Triplasta atrocoxalis Ashmead (Hymenoptera: Figitidae) com 16,2\%.

Palavras-chave: Diptera, Hymenoptera, microhimenópteros, fezes de bovinos
\end{abstract}

\begin{abstract}
This study had the objective of determining the species of microhimenopterous in pupae of Diptera present in cattle dung, collected in the county of Itumbiara, GO, Cachoeira Dourada, GO and Panamá, GO, from January 1998 to June 2004. The dipterous pupae were obtained by the flotation method. They were individually placed in gelatin capsules until the emergence of the flies and/or their parasitoids. The overall prevalence of parasitism was $7.36 \%$. The species more frequent were: Spalangia drosophilae Ashmead (Hymenoptera: Pteromalidae) with 30.8\% e Triplasta atrocoxalis Ashmead (Hymenoptera: Figitidae) with $16.2 \%$.
\end{abstract}

Key words: Diptera, Hymenoptera, microhimenopterous, cattle dung

\footnotetext{
1 Biólogo, Doutor em Ciências Biológicas; Prof. de Zoologia e Parasitologia/Curso de Agronomia. Coordenador de Pesquisa do Instituto Luterano de Ensino Superior de Itumbiara-GO, ILES-ULBRA. E-mail: pesquisa.itb@ulbra.br.

2 Egresso do Curso de Biologia do Instituto Luterano de Ensino Superior de Itumbiara-GO, ILES-ULBRA

3 Aluno de Iniciação Científica do Instituto Luterano de Ensino Superior de Itumbiara-GO, ILES-ULBRA

* Autor para correspondência.
} 


\section{Introdução}

No regime de confinamento e semiconfinamento de animais, o esterco acumulado constitui excelente meio para a criação e desenvolvimento de várias espécies de moscas nos ambientes rurais (ARMITAGE, 1986). Apesar dos excrementos serem retirados periodicamente dos criadouros, sua permanência em esterqueiras favorece o desenvolvimento de uma entomofauna bastante diversificada. Além desses dípteros, também encontram-se outros grupos de artrópodes, como parasitóides, predadores e espécies coprófagas associadas ao esterco bovino (MERRIT; ANDERSON, 1977).

Entre os meios de controle das moscas, os mais utilizados são os inseticidas químicos que podem perder sua eficiência à medida que as populações tornam-se resistentes aos mesmos (SILVEIRA et al., 1989). Além disso, o tratamento com essas substâncias tem ocasionado impacto sobre os inimigos naturais desses insetos (COOK; GERHARDT, 1977), uma vez que os larvicidas não atingem somente à fauna-alvo, mas também acabam prejudicando a fauna de parasitóides e predadores de moscas, que são responsáveis pela redução natural das populações de insetos nocivos. O aparecimento de resistência aos inseticidas justifica a necessidade crescente de implantação de programas alternativos de controle, objetivando o controle de moscas.

O controle alternativo é mais uma questão de conscientização em que se procura mostrar aos produtores a necessidade de retiradas periódicas das fezes acumuladas em esterqueiras (AXTELL; ARENDS, 1990). Estudos indicam que os mais importantes controladores nos criadouros bovinos são os predadores, competidores e parasitóides (GEDEN; STINNER; AXTELL, 1988, SMITH; RUTZ, 1991). A viabilidade do uso de controladores e as vantagens de seu emprego na agricultura e criação de animais são indiscutíveis, em razão de seu baixo custo, facilidade de manuseio, seletividade e a não contaminação ambiental (SILVEIRA et al., 1989).
Esta pesquisa teve como objetivo principal determinar as principais espécies de microhimenópteros parasitóides de Diptera que se desenvolvem em fezes bovinas no Sul do Estado de Goiás.

\section{Material e Métodos}

O experimento em Itumbiara, GO, foi realizado na Fazenda da Faculdade de Agronomia, às margens do rio Paranaíba, a $5 \mathrm{~km}$ do centro de Itumbiara $\left(18^{\circ} 25^{\prime} \mathrm{S}\right.$ e $\left.49^{\circ} 13^{\prime} \mathrm{W}\right), \mathrm{GO}$. A fazenda possui uma área aproximada de 12 alqueires, com um plantel de 45 cabeças de gado bovino leiteiro. As fezes coletadas pertenciam a bovinos resultantes do cruzamento de gado holandês com nelore. As fezes foram expostas em pastagens constituídas de Brachiaria brizantha (Hochst ex. A. Rich). Em Cachoeira Dourada, GO, o experimento foi realizado na Fazenda Boa Vista, distante 20 quilômetros de Cachoeira Dourada $\left(16^{\circ} 40^{\prime} \mathrm{S}\right.$ e $\left.50^{\circ} 40^{\prime} \mathrm{W}\right)$. A fazenda possui uma área aproximada de 114 hectares, com um plantel de 200 cabeças de gado bovino leiteiro. As fezes coletadas pertenciam a bovinos resultantes do cruzamento de gado holandês com nelore. As fezes foram expostas em pastagens constituídas de B. brizantha. No Panamá, o experimento foi realizado na Fazenda Panamá, no município de Panamá, (18 $18^{\circ}$ 'S e $\left.49^{\circ} 21^{\prime} \mathrm{W}\right)$. A fazenda possui uma área aproximada de 114 hectares, com um plantel de 100 cabeças de gado bovino leiteiro. As fezes coletadas pertenciam a bovinos da raça nelore. As fezes foram expostas onde as pastagens eram constituídas também de B. brizantha (Hochst ex. A. Rich).

Quinzenalmente, 10 placas de fezes frescas foram marcadas imediatamente após sua dejeção, com auxílio de estacas de madeira branca $(30 \mathrm{~cm}$ de altura e $5 \mathrm{~cm}$ de espessura), para determinação precisa do tempo entre a dejeção e a coleta, e permaneceram no campo por quinze dias. Sete amostras foram colhidas e levadas ao laboratório do Instituto Luterano de Ensino Superior de Itumbiara, GO, para a extração das pupas pelo método da flotação. Juntamente com as fezes, foram retiradas $5 \mathrm{~cm}$ do substrato situado 
abaixo do local de deposição no solo. As pupas foram retiradas com o auxílio de uma peneira, contadas e individualizadas em cápsulas de gelatina (número 00) até a emergência das moscas e/ou dos parasitóides. Os parasitóides e as moscas emergidos, foram identificados com auxílio de um microscópio estereoscópio, foram conservados em álcool 70\%.

A porcentagem total de parasitismo foi calculada através do número de pupas parasitadas/ número total de pupas coletadas x 100. A porcentagem de parasitismo de cada espécie de parasitóide foi calculada através do número de pupas parasitadas por cada espécie de parasitóide/número total de pupas daquele hospedeiro x 100. O material testemunha foi depositado no Departamento de Biologia do Instituto Luterano de Ensino Superior de
Itumbiara, Goiás. A identificação dos parasitóides foi realizada conforme Legner, Moore e Olton (1976) e Díaz, Gallardo e Marchiori (2000) a dos hospedeiros conforme McAlpine et al. (1981). A hipótese de preferência dos parasitóides pelos seus hospedeiros foi testada pelo Qui-Quadrado.

\section{Resultados e Discussão}

No período de janeiro de 1998 a junho de 2004, em três propriedades rurais no Sul do Estado de Goiás, foram coletados 9657 pupas de Diptera, das quais emergiram 711 parasitóides solitários, evidenciando $7,36 \%$ de porcentagem de parasitismo (Tabela 1).

Tabela 1. Microhimenópteros parasitóides coletados de pupas de dípteros provenientes de fezes de bovinos em três propriedades rurais no Sul de Goiás, no período de maio de 2000 a junho de 2004.

\begin{tabular}{|c|c|c|c|c|}
\hline Grupo Taxonômico & Panamá-GO & Cachoeira Dourada-GO & Itumbiara-GO & Total \\
\hline \multicolumn{5}{|l|}{ Hymenoptera: } \\
\hline \multicolumn{5}{|l|}{ Braconidae: } \\
\hline Gnathopleura quadridentata & 20 & 00 & 00 & 20 \\
\hline \multicolumn{5}{|l|}{ Diapriidae: } \\
\hline Trichopria sp. & 01 & 41 & 46 & 88 \\
\hline \multicolumn{5}{|l|}{ Figitidae: } \\
\hline Kleidotoma nigra & 02 & 00 & 00 & 02 \\
\hline Paraganaspis egeria & 02 & 19 & 32 & 53 \\
\hline Triplasta atrocoxalis & 02 & 41 & 72 & 115 \\
\hline Triplasta coxalis & 08 & 00 & 00 & 08 \\
\hline \multicolumn{5}{|l|}{ Pteromalidae: } \\
\hline Pachycrepoideus vindemmiae & 05 & 00 & 00 & 05 \\
\hline Muscidifurax sp. & 00 & 00 & 02 & 02 \\
\hline Spalangia cameroni & 04 & 01 & 34 & 39 \\
\hline Spalangia drosophilae & 07 & 58 & 154 & 219 \\
\hline Spalangia endius & 02 & 08 & 17 & 27 \\
\hline Spalangia nigra & 07 & 37 & 01 & 45 \\
\hline Spalangia nigroaenea & 18 & 08 & 45 & 71 \\
\hline Spalangia sp. & 00 & 00 & 17 & 17 \\
\hline Total & 78 & 213 & 410 & 711 \\
\hline
\end{tabular}


Pode-se observar que na propriedade do rural Panamá obteve-se uma maior diversidade de parasitóides, quando comparada com as duas outras propriedades. Provavelmente, devido às variações na qualidade e disponibilidade de recursos alimentares ou pelas densidades dos hospedeiros. De maneira geral não houve diferenças em relação às espécies de parasitóides, possivelmente devido às proximidades das propriedades e dos tratos culturais utilizados como: tipo de alimentação, manejo dos animais, utilização de inseticidas no combate aos ectoparasitas e no tratamento dos endoparasitas.

A Spalangia drosophilae Ashmead (Hymenoptera: Pteromalidae) foi a espécie mais coletada com $30,8 \%$ dos indivíduos. S. drosophilae é citada na literatura como parasitóide de pupas de dípteros pequenos das famílias Chloropidae, Drosophilidae, Muscidae, Sarcophagidae e Sepsidae (MARCHIORI; OLIVEIRA; LINHARES, 2001; MARCHIORI, 2002; MARCHIORI et al., 2002). $\mathrm{O}$ fato de utilizar de muitos dípteros, $S$. drosophilae favorece sua permanência, no meio ambiente aumentando o seu potencial como agente no controle biológico de espécies-pragas.

A Triplasta atrocoxalis Ashmead (Hymenoptera: Figitidae) foi a segunda espécie mais coletada com 16,2\% dos indivíduos, em Itumbiara, GO e Cachoeira Dourada, GO, sendo encontrada parasitando Palaeosepsis spp. (Diptera: Sepsidae), Sarcophagula occidua (Fabricius) (Diptera: Sarcophagidae) e Cyrtoneurina pararescita Couri (Diptera: Muscidae) em fezes bovinas (MARCHIORI; SILVA; LINHARES, 2000; MARCHIORI, 2002; MARCHIORI et al., 2002) devido, provavelmente, ao fator sazonalidade apresentada pela espécie.
A porcentagem de parasitismo constatada em Itumbiara, GO, foi de 6,97\%. As espécies de parasitóides mais abundantes foram: $S$. drosophilae com $37,6 \%$ e $T$. atrocoxalis com 17,6\%. A porcentagem de parasitismo constatada em Cachoeira Dourada foi de 6,8\%. As espécies de parasitóides mais abundantes foram: $S$. drosophilae com 27,2\%, Trichopria sp. (Hymenoptera: Diapriidae) e T. atrocoxalis com 19,2\%. A porcentagem de parasitismo observada no Panamá, GO, foi de 12,4\%. As espécies mais freqüentes nas fezes bovinas foram: Gnathopleura quadridentata Wharton (Hymenoptera: Braconidae) com 25,6\% e Spalangia nigroaenea Curtis (Hymenoptera: Pteromalidae) com 23,1\% devido, provavelmente, às densidades dos hospedeiros.

As porcentagens totais de parasitismo em Itumbiara, GO, apresentadas pelos parasitóides Trichopria sp., Paraganaspis egeria Dìaz, Gallardo \& Walsh (Hymenoptera: Figitidae), T. atroxalis, Muscidifurax sp., Spalangia cameroni Perkins, $S$. drosophilae, Spalangia endius Walker, Spalangia nigra Latrielle, S. nigroaenea e Spalangia sp. (Hymenoptera: Pteromalidae) foram, respectivamente, de: $0,78 \%, 0,54 \%, 1,22 \%, 0,03 \%$, 0,58\%, 2,62\%, 0,29\%, 0,02\%, 0,76\% e $0,29 \%$. Portanto, S. drosophilae foi o parasitóide em Itumbiara, GO, que apresentou maior porcentagem de parasitismo em pupas de Diptera (Tabela 2), devido à multiplicidade de ambientes explorados por esta espécie, ela é colocada como promissora em programas de controle, principalmente com a introdução de Haematobia irritans (L.) (Diptera; Muscidae) no Brasil (SILVEIRA et al.,1989). 
Tabela 2. Porcentagem de parasitismo obtido de pupas de dípteros provenientes de fezes bovinas em Itumbiara-GO, de janeiro a dezembro de 1998.

\begin{tabular}{|c|c|c|c|}
\hline Hospedeiro $-\mathrm{n}^{\mathrm{o}}$. de pupas & Parasitóide & $\begin{array}{l}\text { Número de } \\
\text { indivíduos }\end{array}$ & Porcentagem \\
\hline \multirow[t]{7}{*}{ Brontaea quadristigma (720) } & Muscidifurax sp. & 1 & 0,14 \\
\hline & Paraganaspis egeria & 1 & 0,14 \\
\hline & Spalangia cameroni & 18 & 2,50 \\
\hline & Spalangia endius & 8 & 1,11 \\
\hline & Spalangia nigroaenea & 18 & 2,50 \\
\hline & Spalangia sp. & 7 & 1,00 \\
\hline & Trichopria sp. & 3 & 0,42 \\
\hline \multirow[t]{3}{*}{ Brontaea debilis (452) } & Spalangia cameroni & 4 & 0,90 \\
\hline & Spalangia nigroaenea & 4 & 0,90 \\
\hline & Spalangia sp. & 3 & 0,70 \\
\hline \multirow[t]{4}{*}{ Cyrtoneurina pararescita (2362) } & Spalangia cameroni & 1 & 0,04 \\
\hline & Spalangia endius & 2 & 0,08 \\
\hline & Spalangia nigroaenea & 10 & 0,42 \\
\hline & Spalangia sp. & 2 & 0,08 \\
\hline \multirow[t]{8}{*}{ Palaeosepsis spp. (307) } & Muscidifurax sp. & 1 & 0,33 \\
\hline & Spalangia cameroni & 3 & 1,00 \\
\hline & Spalangia drosophilae & 137 & 44,6 \\
\hline & Spalangia endius & 3 & 1,00 \\
\hline & Spalangia nigroaenea & 3 & 1,00 \\
\hline & Spalangia sp. & 3 & 1,00 \\
\hline & Trichopria sp. & 27 & 8,80 \\
\hline & Triplasta atrocoxalis & 67 & 21,8 \\
\hline \multirow[t]{9}{*}{ Sarcophagula occidua (2006) } & Paraganaspis egeria & 31 & 1,55 \\
\hline & Spalangia cameroni & 8 & 0,40 \\
\hline & Spalangia drosophilae & 17 & 0,85 \\
\hline & Spalangia endius & 4 & 0,20 \\
\hline & Spalangia nigra & 1 & 0,05 \\
\hline & Spalangia nigroaenea & 10 & 0,50 \\
\hline & Spalangia sp. & 2 & 0,10 \\
\hline & Trichopria sp. & 8 & 0,40 \\
\hline & Triplasta atrocoxalis & 4 & 0,20 \\
\hline \multirow[t]{2}{*}{ Sphaeroceridae sp. (37) } & Trichopria sp. & 8 & 21,6 \\
\hline & Triplasta atrocoxalis & 1 & 2,70 \\
\hline Total de pupas: 5884 & & & \\
\hline
\end{tabular}

As porcentagens totais de parasitismo em Cachoeira Dourada, GO, apresentadas pelos parasitóides, Trichopria sp. P. egeria, T. atrocoxalis, S. cameroni, S. drosophilae, S. endius, S. nigra e S. nigroaenea foram, respectivamente, de: $1,3 \%$, $0,60 \%, 1,3 \%, 0,03 \%, 1,84 \%, 0,25 \%, 1,18 \%$ e $0,25 \%$.
Portanto, T. atrocoxalis em Cachoeira Dourada, GO, apresentou maior porcentagem de parasitismo em pupas de Diptera (Tabela 3) devido, provavelmente, às variações na qualidade e disponibilidade de recursos alimentares. 
Tabela 3. Porcentagem de parasitismo obtido de pupas de dípteros provenientes de fezes bovinas em Cachoeira Dourada-GO, no período setembro de 2000 a fevereiro de 2001.

\begin{tabular}{|c|c|c|c|}
\hline Hospedeiro - $\mathrm{n}^{0}$. pupas & Parasitóide & $\begin{array}{l}\text { Número de } \\
\text { indivíduos }\end{array}$ & Porcentagem \\
\hline \multirow[t]{2}{*}{ Archisepsis scabra (129) } & Spalangia drosophilae & 06 & 4,65 \\
\hline & Triplasta atrocoxalis & 08 & 6,20 \\
\hline Brontaea debilis (95) & Spalangia nigra & 01 & 1,05 \\
\hline Coproica sp. (340) & Trichopria sp. & 30 & 8,82 \\
\hline \multirow[t]{3}{*}{ Cyrtoneurina pararescita $(302)$} & Paraganaspis egeria & 02 & 0,66 \\
\hline & Spalangia endius & 03 & 0,99 \\
\hline & Spalangia nigroaenea & 02 & 0,66 \\
\hline \multirow[t]{2}{*}{ Haematobia irritans (28) } & Paraganaspis egeria & 01 & 3,57 \\
\hline & Trichopria $\mathrm{sp}$. & 05 & 17,9 \\
\hline Musca domestica (227) & Spalangia nigroaenea & 03 & 1,32 \\
\hline \multirow[t]{3}{*}{ Palaeosepsis spp. (1197) } & Spalangia drosophilae & 42 & 3,50 \\
\hline & Trichopria sp. & 04 & 0,33 \\
\hline & Triplasta atrocoxalis & 32 & 2,67 \\
\hline \multirow[t]{8}{*}{ Sarcophagula occidua (827) } & Paraganaspis egeria & 16 & 1,93 \\
\hline & Spalangia cameroni & 01 & 0,12 \\
\hline & Spalangia drosophilae & 10 & 1,21 \\
\hline & Spalangia endius & 05 & 0,60 \\
\hline & Spalangia nigra & 36 & 4,35 \\
\hline & Spalangia nigroaenea & 03 & 0,36 \\
\hline & Trichopria sp. & 02 & 0,24 \\
\hline & Triplasta atrocoxalis & 01 & 0,12 \\
\hline Total de pupas: 3145 & & & \\
\hline
\end{tabular}

As porcentagens totais de parasitismo apresentadas pelos parasitóides, Gnathopleura quadridentata, Trichopria sp., Kleidotoma nigra (Hartig) (Hymenoptera: Figitidae), P. egeria T. atrocoxalis, Triplasta coxalis Ashmead (Hymenoptera: Figitidae), Pachycrepoideus vindemmiae (Rondani) (Hymenoptera: Pteromalidae), S. cameroni, S. drosophilae, S. endius, S. nigra,
S. nigroaenea foram, respectivamente, de: $3,2 \%$, $0,16 \%, 0,32 \%, 0,32 \%, 0,32 \%, 1,27 \%, 0,79 \%, 0,64 \%$, $1,11 \%, 0,32 \%, 1,11 \%$ e 2,87. Gnathopleura quadridentata no município do Panamá, GO, apresentou maior porcentagem de parasitismo em pupas de Diptera (Tabela 4) devido, provavelmente, a sua capacidade de busca. 
Tabela 4. Porcentagem de parasitismo obtido de pupas de dípteros provenientes de fezes bovinas no município dedo Panamá-GO, Goiás, no período de maio de 2003 a junho de 2004.

\begin{tabular}{|c|c|c|c|}
\hline Hospedeiro - $\mathrm{n}^{\circ}$. pupas & Parasitóide & $\begin{array}{l}\text { Número de } \\
\text { indivíduos }\end{array}$ & Porcentagem \\
\hline Archisepsis scabra (40) & Spalangia drosophilae & 04 & 10,0 \\
\hline \multirow[t]{2}{*}{ Brontaea debilis (56) } & Spalangia cameroni & 01 & 1,79 \\
\hline & Spalangia nigroaenea & 02 & 3,57 \\
\hline \multirow[t]{5}{*}{ Brontaea quadristigma (49) } & Kleidotoma nigra & 02 & 4,08 \\
\hline & Spalangia cameroni & 01 & 2,04 \\
\hline & Spalangia drosophilae & 01 & 2,04 \\
\hline & Spalangia. endius & 01 & 2,04 \\
\hline & Spalangia nigroaenea & 05 & 10,2 \\
\hline Cyrtoneurina pararescita (151) & Spalangia nigra & 03 & 1,99 \\
\hline Chrysomya megacephala (51) & $\begin{array}{l}\text { Spalangia nigroaenea } \\
\text { ausente }\end{array}$ & 05 & 3,31 \\
\hline Musca domestica (10) & Spalangia cameroni & 01 & 10,0 \\
\hline Oxysarcodexia thornax (70) & Gnathopleura quadridentata & 20 & 28,6 \\
\hline \multirow[t]{6}{*}{ Palaeosepsis sp. (107) } & Paraganaspis egeria & 02 & 1,87 \\
\hline & Spalangia drosophilae & 02 & 1,87 \\
\hline & Spalangia endius & 01 & 0,93 \\
\hline & Triplasta atrocoxalis & 02 & 1,87 \\
\hline & Triplasta coxalis & 08 & 7,48 \\
\hline & Trichopria sp. & 01 & 0,93 \\
\hline \multirow[t]{4}{*}{ Ravinia belforti (63) } & Pachycrepoideus vindemmiae & 05 & 7,94 \\
\hline & Spalangia cameroni & 01 & 1,59 \\
\hline & Spalangia nigra & 04 & 6,35 \\
\hline & $\begin{array}{l}\text { Spalangia nigroaenea } \\
\text { ausente }\end{array}$ & 06 & 9,52 \\
\hline Total de pupas: 628 & & 78 & 14,0 \\
\hline
\end{tabular}

Com relação à preferência dos parasitóides pelos seus hospedeiros em Itumbiara, $\mathrm{GO}$, verificou-se que: T. atrocoxalis apresentou preferência por pupas de Palaeosepsis spp. (Diptera: Sepsidae) e P. egeria por pupas de $S$. occidua $\left(\mathrm{X}^{2}=5,49\right.$ : $\mathrm{GL}=1$ : $\left.\mathrm{P}<0.005\right)$. $S$. cameroni apresentou preferência por pupas de Brontaea quadristigma (Thomson) (Diptera: Muscidae) e S. occidua; S. drosophilae por pupas de Palaeosepsis spp.; $S$. endius por pupas de $B$. quadristigma, $C$. pararescita e $S$. occidua; $S$. nigroaenea por pupas de B. quadristigma, $C$. pararescita e $S$. occidua; Trichopria sp. apresentou preferência por pupas de $S$. occidua e Sphaeroceridae sp. (Diptera: Sphaeroceridae) $\left(\mathrm{X}^{2}=\right.$ $61,9 ; \mathrm{GL}=16 ; \mathrm{P}<0.05)$.
Em Cachoeira Dourada, verificou que: $S$. drosophilae associou-se a pupas de Palaeosepsis spp. e Archisepsis scabra (Loew) (Diptera: Sepsidae); $S$. endius associou-se a pupas de $C$. pararescita e $S$. occidua; $S$. nigra associou-se a pupas de $S$. occidua; S. nigroaenea associou-se a pupas de C. pararescita, Musca domestica L. (Diptera: Muscidae) e S. occidua; P egeria associouse a pupas de $H$. irritans e $S$. occidua; $T$. atrocoxalis associou-se a pupas de A. scabra e Palaeosepsis spp. e Trichopria sp. associou-se a pupas de H. irritans e Coproica sp. (Diptera: Sphaeroceridae) $\left(\mathrm{X}^{2}=419,24 ; \mathrm{GL}=49 ; \mathrm{P}<0,05\right)$. 
Marchiori, C. H.; Silva Filho, O. M.; Borges, M. P.

Com relação à preferência dos parasitóides pelos seus hospedeiros em fezes bovinas no município do Panamá, GO, verificou-se que, G. quadridentata apresentou preferência por Oxysarcodexia thornax (Wiedemann) (Diptera: Sarcophagidae); K. nigra mostrou preferência por B. quadristigma; $P$. vindemmiae apresentou preferência por Palaeosepsis spp.: S. cameroni apresentou preferência por $B$. debilis e $M$. domestica; $S$. drosophilae apresentou preferência por A. scabra e $M$. domestica; $S$. endius apresentou preferência por B. quadristigma e Palaeosepsis spp.; S. nigra apresentou preferência por $C$. pararescita e Ravinia belforti (Prado \& Fonseca) (Diptera: Sarcophagidae); $S$. nigroaenea apresentou preferência por B. debilis, B. quadristigma, $C$. pararescita e $R$. belforti $\left(\mathrm{X}^{2}=715,24\right.$; GL: 77; $\mathrm{P}<0.05)$.

Aumenta-se com este estudo a bioecologia e a distribuição geográfica dos microhimenópteros parasitóides de Díptera que se desenvolvem em fezes de gado bovino no Brasil.

\section{Referências}

ARMITAGE, D.M. Population changes of four species of insects (Coleoptera: Diptera) in three deep poultry houses. Entomologist' S Monthly Magazine, Oxford, v.122, p.7577, 1986.

AXTELL, R.C.; ARENDS, J.J. Ecology and management of arthropod pests of poultry. Annual Review of Entomology, Stanford, v.35, p.101-126, 1990.

COOK, C.W.; GERHARDT, R.R. Selective mortality of insects in manure from cattle fed rabon and dimilin. Environmental Entomology, College Park, v.6, p.46-48, 1977.

DÍAZ, N.B.; GALLARDO, F.E.; MARCHIORI, C.H. Cynipoidea parasitoids of dung-flies in Brazil. II (Insecta: Hymenoptera). Anais da Sociedade Entomologica do Brasil, Jaboticabal, v.29, n.3, p.469-474, 2000.

GEDEN, C.J.; STINNER, R.E; AXTELL, R.C. Predation by predators of the house fly in poultry manure: effects of predator density, feeding history, interspecific interference, and field conditions. Environmental Entomology, College Park, v.17, p.320-329, 1988.
LEGNER, E.E; MOORE, I.; OLTON, G.S. Tabular keys \& biological notes to commom parasitoids of synanthropic Diptera breeding in accumulated animal wastes. Entomological News, Philadelphia, v.87, p.113-144, 1976.

MARCHIORI, C.H.; SILVA, CG.; LINHARES, A.X. Primeira ocorrência de Triplasta atrocoxalis Ashmead (Hymenoptera: Eucoilidae) em pupas de Cyrtoneurina pararescita Couri (Diptera: Muscidae) em currais de bovinos no Brasil. Arquivo Brasileiro de Medicina Veterinaria e Zootecnia, Belo Horizonte, v.52, n.1, p.3940,2000.

MARCHIORI, C.H.; OLIVEIRA, A.T.; LINHARES, A.X. Artrópodes associados a massas fecais bovinas no Sul do Estado de Goiás. Neotropical Entomology, Londrina, v.30,p.19-24, 2001

MARCHIORI, C.H. Microhimenópteros parasitóides de moscas em esterco bovino em Cachoeira Dourada, Goiás, Brasil. Entomologia y Vectores, Rio de Janeiro, v.9, p.365374, 2002.

MARCHIORI, C.H.; PEREIRA, L.A.; SILVA FILHO, O.M.; RIBEIRO, L.C.S. Paraganaspis egeria Díaz, Gallardo \& Wash (Hymenoptera: Figitidae: Eucoilinae) as potential agent in the biocontrol of muscoid dipterous collected in several substracts in Itumbiara, Goiás, Brazil. Arquivo Brasileiro de Medicina Veterinaria e Zootecnia, Belo Horizonte, v.54, n. 6, p.662-664, 2002.

McALPINE, J.E.; PETERSON, B.V.; SHEWEELL, G.E.; TESKEY, H.J.; VOCKROTH, J.R.; WOOD, D. M. Manual of neartic Diptera. Otawa: Research Branche Agriculture Canada, 1981.1332 p.

MERRIT, R.W; ANDERSON, J.R. The effects of different pasture and rangeland ecosystems on the annual dynamics of insects in cattle droppins. Hilgardi, Berkeley, v.45, p.31-71, 1977.

SILVEIRA, G.A.R.; MADEIRA, N.G.; AZEREDO, E.; PAVAN, C. Levantamento de microhimenópteros parasitóides de dípteros de importância medico-veterinária no Brasil. Memória do Instituto Oswaldo Cruz, Rio de Janeiro, v.84, p.505-510, 1989.

SMITH, L.; RUTZ, D.A. Seasonal and relative abundance of hymenopterous parasitoids attacking house fly pupae at dairy farms in Central New York. Environmental Entomology, College Park, v.20, p.661-668, 1991. 